\title{
Los ambientes de aprendizaje en el aula
}

\author{
Learning environments in the classroom
}

James Frank Becerra Martineq+1

Bibiana Quiroga Perezs

Oscar Leonardo Cardenas Forero*1

Rosa Inés Pedrero $x_{4}$

Sonia Milena Uribe Carzón*1

Maria Anais Moncada Rodrigue*5

Maria Gilma Acosta Rodrigurez +1

Cristina Behrentz Pfal $\$ 6$

Edith Constanza Negrete Soler*2

Libia Mireya Garzon Moren@

Resumen

El colectivo "Maestros en colectivo: construyendo y deconstruyendo miradas y sentidos de los ambientes de aprendizaje en el aula", recoge vivencias propias y ajenas, tejidas con hilos invisibles entre los estudiantes y maestros, la actividad y el contexto, y allega elementos que hacen posible caracterizar el ambiente de aprendizaje en el aula y el enriquecer el saber pedagógico, en tanto se reconoce al docente como constructor, en la reflexión de su práctica docente.

\section{Palabras dave}

Ambientes de aprendizaje, aula, actividad, contexto, sujetos, conocimiento.
Summary

"Teachers in collective: building and rebuilding views and senses of learning environments in the classroom" is a group that collects the experiences of these teachers and of others, woven with invisible threads between students and teachers, and between the activity and the context. It also provides elements that make possible the characterization of the classroom learning environment and the enrichment of pedagogical knowledge. Therefore, the teacher is recognized as a builder, in the reflection of his/her teaching practice.

Kor words

Learning environments, classroom, activity, context, subjects, knowledge.

* Este escrito hace parte de las reflexiones adelantadas en la investigación "Maestros en colectivo: construvendo y deconstruyendo miradas y sentidos de los ambientes de aprendizaje en el aula", financiada por IDEP como Experiencia de Frontera del Laboratorio Pedagógico 2004-2005 y la Corporación Escuela Pedagógica Experimental (CEPE).

*1 IED Entre Nubes Suroriental.

$* 2$ IED Antonio Baraya.

*3 IED Toscana Lisboa.

*4 Universidad Pedagógica Nacional.

*5. IED Gustavo Restrepo

*6 IED José Martí.

*7 Gimnasio Sabios del Bosque.

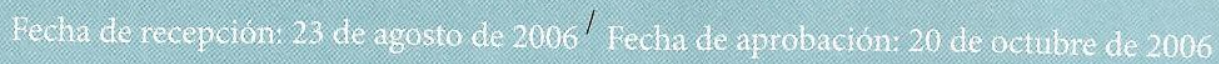




\section{Presentación}

\section{Los maestros}

que en la actualidad participan en esta experiencia venían desarrollando propuestas que emergían de las inquietudes e intereses particulares de los docentes frente a situaciones escolares. Propuestas en las que se encuentran elementos para enriquecer la práctica pedagógica y superar el solipsismo de los maestros.

La investigación Un acercamiento a la escuela en los niños del Centro Educativo Distrital (CED) Santa Rita Suroriental, jornada tarde ${ }^{1}$, socializada en el V Simposio sobre Enseñanza de las Ciencias en la Educación Básica y Media ${ }^{2}$ y en el II Encuentro de Maestros y Comunicación de Experiencias ${ }^{3}$, pone en evidencia la ambivalencia entre los imaginarios que tienen los niños y sus maestros respecto a la concepción de escuela: "Espacio lleno de salones, baños, pupitres, profesores, rejas, ventanas, niños y cuadernos, en donde se viene a aprender a sumar, a restar, a multiplicar, a dividir, a leer y escribir; pero también en donde se puede jugar, a veces a escondidas del profesor" (opinión de los estudiantes).

El registro en diarios de campo durante un año y su sistematización dieron cuenta de una serie de afirmaciones sobre la realidad del aula: el docente de una u otra manera asume el reto del conocimiento escolar desde la información, lo que ocasiona que en el estudiante se evidencien solo determinadas habilidades y destrezas, que permean casi todas las clases. La validación del conocimiento se da en tanto la información que se espera debe ser similar a la que hace circular el maestro. El educador es en gran parte responsable de que la escuela, el conocimiento y el sujeto solo sean valorados desde lo aritmético y lo lecto-escritural. Esto condujo, en el año 2000, al planteamiento de un problema que ha servido de ruta en el diseño y aplicación de propuestas pedagógicas desde los ambientes de aprendizaje: en el currículo en gran medida se privilegia en las diversas actividades en el aula la aritmética y la lectoescritura

\footnotetext{
1 Investigación que se realizó durante 1999, con el apoyo de la Corporación Escuela Pedagógica Experimental (CEPE), a cargo de los docentes James Frank Becerra M., Óscar L. Cárdenas F., Olga C. Ajiaco A. y Mario J. Puche C.

2 Evento organizado por la CEPE, el grupo de Física y Cultura de la Universidad Pedagógica Nacional y la Red de Cualificación de Docentes en Ejercicio, RED-CEE. Bogotá 18, 19 y 20 de agosto de 2000.

3 Encuentro realizado por la CEPE. Bogotá, 4 y 5 de noviembre de 2000.
}

como únicos aspectos relevantes en la formación de los estudiantes, olvidando que el sujeto en su experiencia aborda las situaciones desde múltiples formas.

En los años 2000 y 2001 se implementó la propuesta "El estudio de las inteligencias múltiples: una posibilidad de diseñar currículo desde el aula" ${ }^{4}$, donde se buscaban estrategias para ir mas allá de ciertas habilidades y destrezas netamente escolares. La forma como la escuela hace sentir a sus estudiantes frente al conocimiento los lleva a creer que solo son válidos los aspectos de la vida escolar que respondan a unas temáticas específicas: lectoescritura y las destrezas matemáticas (operaciones básicas). Durante el proceso de escolaridad del individuo (al menos en la básica primaria) se es inteligente solo en la medida en que se cumplan unas expectativas o tareas escolares; las habilidades deportivas, artísticas y emocionales no son consideradas como una manifestación de la inteligencia.

Las ponencias “Quehacer matemático en la escuela: una reflexión desde el enfoque de maestros no matemáti$\cos ^{\prime} ;$ “ ¿Qué tiene para ofrecer la clase de matemáticas al desarrollo del pensamiento?"5 y "Pensar matemáticamente: una manera distinta de enfocar el ambiente matemático en la escuela" ${ }^{\prime}$, dan cuenta de que al mediar situaciones dentro del ambiente de aprendizaje de la matemática por desafíos, problemas y tensiones, es decir, que el pensar circule en su interior, esta perderá su carácter de aburrida y estática, para dar paso a un universo en el que se le presentan a los niños una serie de situaciones y actividades que favorecen en gran medida la constitución del pensamiento matemático.

En el CED Luis López de Mesa se gestó en el año 2000 la experiencia de aula "Aprender caminando y observando: una experiencia significativa"7. En este trabajo se asumen las salidas pedagógicas como prácticas es-

\footnotetext{
4 Propuesta de los docentes James Frank Becerra M. y Óscar L. Cárdenas F., socializada en el I Encuentro de Maestros "De la insularidad a la práctica de lo colectivo", organizado por la RED-CEE en el año 2001 y en la Convocatoria Fundación Ford y Expedición Pedagógica Nacional en el año 2002.

5 Ponencias socializadas en el III Encuentro de Maestros y Comunicación de Experiencias organizado por la CEPE y en el $V$ Simposio de la Enseñanza de las Ciencias en el nivel Básico y Medio, Universidad Pedagógica Nacional-EPE, junio de 2001. Bogotá.

6 Ponencia presentada en el VII Foro Educativo Local (Localidad San Cristóbal) y en el VII Foro Educativo Distrital. Bogotá. 2002.

7 Experiencia de la maestra María G. Acosta R., VIII Foro Educativo Local (Localidad Rafael Uribe Uribe) y en el VIII Foro Educativo Distrital "De la curiosidad a la actitud científica". 2003.
} 
colares que contribuyen en los procesos de aprendizaje significativo desde la investigación-acción participativa. Bajo esta perspectiva se deja entrever que el ambiente de aprendizaje trasciende el salón de clase y puede ser ubicado en relación con el entorno. De esta forma se abren posibilidades para caracterizar un ambiente de aprendizaje desde el conocimiento, los sujetos, los escenarios y las relaciones que entre ellos se establecen.

En el CED Antonio Baraya la propuesta "Enseñanzaaprendizaje por investigación: Resolución de preguntas problema" ${ }^{8}$ desde 2001, desde la implementación de una estrategia didáctica cuyo instrumento fundamental es la $\vee$ heurística, busca el permitir el reconocimiento en la construcción del conocimiento.

Estas propuestas y experiencias dan cuenta de las inquietudes e intereses que son comunes para algunos maestros del CED Santa Rita Suroriental (sede D de la actual IED Entre Nubes Suroriental), CED Baraya (sede A de la actual IED Antonio Baraya) y CED Luis López de Mesa (sede A de la actual IED José Martí), particularmente la reflexión en torno a los ambientes de aprendizaje, la sistematización y la socialización de sus prácticas pedagógicas como una estrategia de calificación en diferentes espacios académicos.

En estos encuentros y participaciones los maestros ubican la pertinencia de conformar un grupo, inquietud que se fortalece a partir de reuniones, talleres, conversatorios, lectura de artículos y documentos y socialización de las vivencias en el aula. Dinámica que adquiere sentido por el nivel de profundidad en las reflexiones, producción de escritos (ponencias, diseño de actividades, relatorías, etc.), constituyéndose de esta manera un colectivo cuyo centro de trabajo es la discusión sobre los ambientes de aprendizaje en el aula y la búsqueda de la validación del saber pedagógico.

En la actualidad se ha consolidado el colectivo con maestros de diferentes instituciones, que se encuentra desarrollando experiencias en sus respectivas aulas, reflexionando y participando en el Seminario de Formación Permanente. Estas nuevas propuestas que enriquecen la mirada del maestro, la práctica de cada uno y los discursos sobre la escuela, la educación, los

8 Propuesta de la docente Edith Negrete S., socializada en el VIII Foro Educativo Local (Localidad Rafael Uribe Uribe) y en el VIII Foro Educativo Distrital "De la curiosidad a la actitud científica". 2003. aprendizajes y los ambientes de aprendizaje en el aula, son las siguientes:

En la IED Toscana-Lisboa sede C la docente Ana Bibiana Quiroga aborda la estrategia metodológica aprenderjugando a través del desarrollo de actividades lúdicas como sociodramas, presentaciones, bailes, cantos, desfiles, pintura y artes plásticas, las cuales han permitido que los estudiantes las consideren como posibilidades de expresión natural de lo que sienten y piensan.

En la IED José Martí la docente Cristina Behrentz aborda ejes temáticos transversales para desarrollar habilidades comunicativas, privilegiando los procesos de escritura y lectura frente a los contenidos. Se fundamenta en la lectura compartida alrededor de la pregunta: ¿De qué manera se puede incentivar el desarrollo de las habilidades comunicativas en niños?

En la sede B de la IED Entre Nubes Suroriental la docente Sonia Uribe propone el juego del ajedrez como una estrategia didáctica que busca desarrollar el pensamiento y mejorar las relaciones de convivencia de los niños. Se concibe este juego como un valioso instrumento de motivación y reconocimiento del otro, en términos de aprendizaje cooperativo.

La profesora Anais Moncada plantea en la IED Gustavo Restrepo propuestas de aprendizaje en el área de tecnología, orientadas a propiciar herramientas básicas que permitan a los egresados desempeñarse tanto en lo laboral como en lo académico mediante el diseño y la elaboración de modelos y artefactos.

En el Gimnasio Sabios del Bosque la docente Libia M. Garzón M. aborda la propuesta la sinfonía del saber a través de la música como instrumento para estimular y desarrollar el aprendizaje de los niños de la escuela inicial.

\section{Miradas y sentidos de los ambientes de aprendizaje en el aula}

En el ámbito pedagógico se plantean diversas formas de comprender los ambientes de aprendizaje. Cada una de estas perspectivas se convierte en el fundamento para enriquecer, deconstruir y construir miradas y sentidos que sobre ellos se proponen.

En la experiencia pedagógica "El ambiente de aprendizaje desde la vivencia de las inteligencias múltiples" el 
ambiente de aprendizaje se entiende como el encuentro de intersubjetividades, no casual o al azar, sino intencionado y propiciado especialmente por el maestro en su deber ser. La intersubjetividad está dada por relaciones sociales en el reconocimiento del otro (maestro, estudiante, padres de familia), el encuentro está mediado por el tiempo en el que se presenta la interacción con el otro, en un escenario: aula, escuela, humedal, plaza de mercado, museo.

"Ambiente de aprendizaje significativo: el reto de problematizar". Este proyecto considera que el ambiente de aprendizaje, particularmente de las ciencias naturales, hace referencia a saberes cotidianos, escolares y disciplinares de los estudiantes en un espacio físico donde se gestan las interacciones entre los diferentes actores, contextos, representados y vividos por sujetos que dan cuenta de las características de una comunidad.

En la experiencia "El aula, un ambiente lúdico de aprendizaje" el ambiente de aprendizaje se concibe como un espacio dinámico e interactivo donde se potencian múltiples saberes y conocimientos capaces de incidir en los procesos de la condición humana. El encuentro con el otro resulta determinante para configurar un ambiente que trasciende no solo los modos de conocer y aprender sino ante todo de actuar.

Para la "Sinfonía del saber" el ambiente de aprendizaje se expresa como un conjunto armonioso, de diferentes elementos, escenarios, contextos, objetivos, contenidos, actividades y sujetos, entre otros, que se convierten en espacio educativo generador de cambio. Este se presenta en cuanto hay un papel dinámico del maestro, la interacción con sus estudiantes y la organización en el aula.

En cuanto a "Lectura compartida como estrategia para el desarrollo de las habilidades comunicativas", el ambiente de aprendizaje es la conjugación de lo físico y sicológico para desarrollar la labor de enseñanza-aprendizaje. El ambiente de aprendizaje se crea a partir de la actitud y disposición del docente y sus estudiantes en el lugar donde ocurre la práctica pedagógica.

"Interactuando y aprendiendo jugando ajedrez" es un proyecto en donde el ambiente de aprendizaje es concebido como un espacio de compartir con el otro a través del juego y de las vivencias de los saberes que se tienen.
Al observar cada una de las miradas que desde estos proyectos de aula se tienen sobre los ambientes de aprendizaje en el aula, se encuentran ciertos elementos comunes que se convierten en el fundamento para la construcción de la propuesta colectiva. El ambiente no es algo dado, estático sino que se constituye y caracteriza en la interacción e interrelación entre los sujetos o actores (maestros y estudiantes) reconocidos como seres integrales, que no es posible homogeneizar ni en gustos ni en intereses ni en el saber y que por lo tanto no son unidimensionales; la actividad que corresponde a las acciones y el hacer de los sujetos donde se genera una serie de conocimientos: pedagógicos para el maestro y escolares para sus estudiantes, y el contexto como las condiciones políticas, históricas, culturales e ideológicas de la sociedad en un espacio-tiempo particular. Los ambientes de aprendizaje en el aula que se configuran son intencionados y a la vez toman forma dependiendo de lo que emerge de estas interacciones e interrelaciones, por lo tanto, ellos se van transformando, enriqueciendo permanentemente y constituyendo un entramado de significaciones y sentidos para aquellos que los vivencian.

Cada una de estas apreciaciones enriquecidas por las reflexiones, producciones y discusiones fundamenta una propuesta que ha sido llamada la metáfora de la red sobre ambientes de aprendizaje en el aula.

En cada una de estas interacciones se leen maneras de ser maestro en sus prácticas pedagógicas, de ser sujeto en interrelación con el otro, de aprender, de conocer, de evaluar, de hacer escuela y de producir saber y conocimiento. Al transformarse el ambiente, en tanto se vivencian otras maneras de entender $y$ ver al sujeto, de asumir, proponer $y$ hacer la actividad y tener en cuenta el contexto, se asume la escuela desde un enfoque que trasciende lo que usualmente se ha venido haciendo: centrar el trabajo en la información y pensar que todos aprenden igual.

Los ambientes de aprendizaje en el aula tienen que ver con una cultura que se hace evidente en las instituciones educativas cuando se relacionan formas de ser, expectativas y fines que confluyen, donde se potencian acciones para la formación de sujetos. Hoy más que nunca se tiene que pensar la escuela como un escenario donde no solamente hay un proceso de conocimiento en un ámbito del saber, sino que lleve a 
los sujetos a la regulación, a ser partícipes activos con su saber en las comunidades a las cuales pertenecen. En esta propuesta hay maneras de asumir el sujeto, el conocimiento, la evaluación, el contexto, la actividad y las salidas pedagógicas (como otros escenarios diferentes al salón de clase).

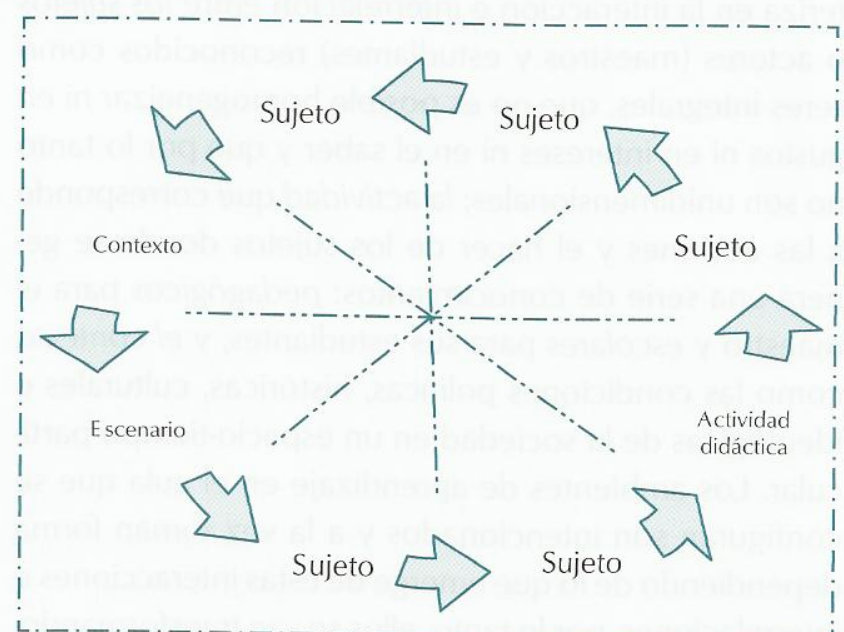

Figura 1. La metáfora de la red, una forma de comprender los ambientes de aprendizaje en el aula

\section{El sujeto}

Desde esta propuesta, el sujeto es reconocido desde una serie de aspectos que se alejan del carácter "unidimensional" que en gran medida ha servido para valorarlo. "Hoy existen numerosos argumentos para sostener que no es posible homogenizar al individuo, ni en sus gustos e intereses, ni en la talla de su ropa, ni en sus capacidades mentales, ni en los ritmos que determinan sus formas de interacción con el mundo, con el saber, con sus iguales o consigo mismo" (Segura, 1999). Ese sujeto puede ser asumido como un sujeto dado, es decir como una persona con una historia, una forma de comportarse, con experiencias de conocimiento, valores, emociones y pensamientos y como un sujeto no dado que se configura, emerge y aparece en la interacción que se establece con los otros, y es allí donde aparece su historia, sus experiencias de conocimiento, valores, emociones, pensamientos y comportamientos.

\section{El conocimiento}

Desde esta perspectiva, el conocimiento es entendido como una construcción que se hace en la interacción colectiva (como en muchos estudios lo ha mencionado Vigotsky), por lo cual, este no se construye en el solipsismo, pues se necesita del otro para validarlo y, por supuesto, para darlo a conocer. En este sentido, esta imagen se aparta de la concepción usual que lo considera como "(...) un conjunto de resultados, esto es, como un catálogo de leyes, teorías, principios y algoritmos (...)" (Segura, 1999); al mismo tiempo, lleva a diferenciar en el ámbito escolar entre lo que significan la información y el conocimiento. La información hace referencia a las generalizaciones ("afirmaciones fuera de contexto") y el conocimiento se refiere a lo particular y específico. La información (sin desconocer su valor en la construcción de conocimiento) se transforma en conocimiento cuando el estudiante la utiliza para solucionar una situación problémica, explicar y comprender un fenómeno social y natural.

El conocimiento se relaciona con el intento por aportar posibles soluciones a problemas genuinos (emergencia de inquietudes), con la resolución de conflictos, con el desarrollo de proyectos, con la necesidad de responder a interrogantes, con la explicación y comprensiones de fenómenos sociales y naturales, con el asumir actitudes morales, investigativas, personales, críticas y reflexivas.

\section{La actividad}

Las actividades que se plantean en los ambientes de aprendizaje en el aula se diseñan bajo la forma de una pregunta, un problema (definido como una "situación abierta expresada a través de enunciados interesantes y sencillos, capaz de generar descubrimientos mediante la aplicación de heurísticas apropiadas que permitan al estudiante plantear ejemplos, contraejemplos, hacer verificaciones y proponer conjeturas" [Lakatos] o como una "herramienta a través de la cual el estudiante es colocado en situación de acción y transformación" [Maza, C.], un taller ${ }^{9}$, un desafío, un juego, un interrogante, un conflic to histórico-social, un proyecto según los intereses y expectativas de los estudiantes (relacionado con un tema, una idea o una cuestión de la vida cotidiana).

Simultáneamente se proponen opciones de carácter metodológico y estrategias educativas, por ejemplo, trabajo en equipo, búsquedas particulares, resolución de problemas, debates e interlocuciones, diseño y cons-

\footnotetext{
Significa un "(...) sistema de enseñanza/aprendizaje [que hace referencia a] un lugar donde se trabaja, se elabora y se transforma algo para ser utilizado. Se trata, pues, de un aprender haciendo". (Ander-Egg, Ezequiel. Capítulo IV. "Programación de aula". En: La planificación educativa. Conceptos, métodos estrategias y técnicas para educadores. Buenos Aires: Editorial Magisterio del Río de la Plata, 6ª ed., 1996, p. 213.
} 
trucción de modelos explicativos, diálogos constantes. De igual manera, se eligen para la materialización de las actividades.

\section{La evaluación}

En esta propuesta, se exige a la evaluación una transformación desde su concepción usual a otra que contemple elementos conducentes a favorecer la participación y el reconocimiento de potencialidades, expectativas e inquietudes humanas. En tal sentido, su énfasis está en los procesos, presentes en todos los eventos ocurrentes en los ambientes de aprendizaje en el aula, es decir, ha de ser utilizada para el mejoramiento y optimización, no en términos de controlar o medir, sino para orientar, guiar y potenciar.

\section{El contexto}

Estudios e investigaciones en el campo de la educación y la pedagogía suelen afirmar, entre otros aspectos, que para que la educación sea pertinente y adquiera sentido, tanto para quienes se involucran en este proceso como para los intereses, necesidades y expectativas del proyecto colectivo de país y nación, es fundamental ubicarla en un contexto, es decir, contextualizarla. Sin embargo, ¿qué es lo que se está denominando contexto?, más aún, cuando en algunas ocasiones suelen atribuírsele como sinónimos conceptos como el de escenario.

Cuando hablamos de escenario, se hace referencia a un lugar específico, a un espacio físico determinado en el que interactúan maestros y estudiantes y en el que se propicia un ambiente de aprendizaje. Algunos ejemplos de escenario son el salón de clase, los museos, la biblioteca, la plaza de mercado, el barrio y la ciudad, los cuales pueden estar sujetos a las intenciones del maestro.

No obstante, al hablar de contexto la cuestión es de por sí más compleja, puesto que no solo involucra los escenarios mismos sino un sinnúmero de elementos - de carácter histórico, social, económico, cultural, tecnológico, científico y político- que condicionan y se retroalimentan con el actuar de los grupos humanos. Por ejemplo, algunos autores como Quintana, Pérez y Morin lo comprenden como:

Para Quintana (1999), el contexto está constituido principalmente por dos aspectos:
Primero, el espacio físico, geográfico o espacial, donde el individuo ejecuta las acciones que permiten inferir las competencias que está utilizando en un momento dado, recalcando que un individuo puede desempeñarse adecuadamente en un contexto, pero no desempeñarse adecuadamente en otro contexto. Y segundo, el contenido de ese contexto, [es decir], lo que también se ha llamado el texto, que son las redes de significado o redes simbólicas de saberes que establecen los sujetos que comparten el espacio físico, [el cual] le da contenido y sustancia a las relaciones que se establecen al interior de un grupo en un contexto específico.

Estas redes hacen referencia al espacio cultural de un grupo, en donde se encuentran parámetros históricos, religiosos, sicológicos, laborales, de aprendizaje, ideológicos, etc., que les dan identidad y pertenencia a los individuos de esa colectividad. El mismo autor menciona una tercera idea acerca de este aspecto, la cual hace referencia a las disciplinas del conocimiento y sus diferentes abordajes, teorías o postulados. Es decir, el contexto que se relaciona con las diferentes concepciones que se desprenden de una disciplina del saber.

A su vez, Morin (1999) sostiene que al estar inmersos el conocimiento y la educación en la era planetaria, "se necesita situar todo en contexto y en la complejidad planetaria". El contexto actual es el mundo mismo, uno de los problemas universales de todo ciudadano del nuevo milenio es saber cómo percibir y concebir el contexto. Lo anterior significa que para que un conocimiento y la educación misma sean pertinentes hay que ubicarlos en contexto y dejar de considerarlos como elementos aislados.

\section{Las variables de transformación en los ambientes de aprendizaje en el aula}

Considerando que la clase es nuestra primera herramienta y el marco de referencia para leer las distintas formas de concebir los ambientes de aprendizaje en el aula, se proponen categorías, indicadores cualitativos y variables de transformación que permiten abordar de manera descriptiva la lectura de los ambientes de aprendizaje en el aula. Se presenta a continuación una muestra de los registros que evidencian la realidad escolar desde dos puntos de 


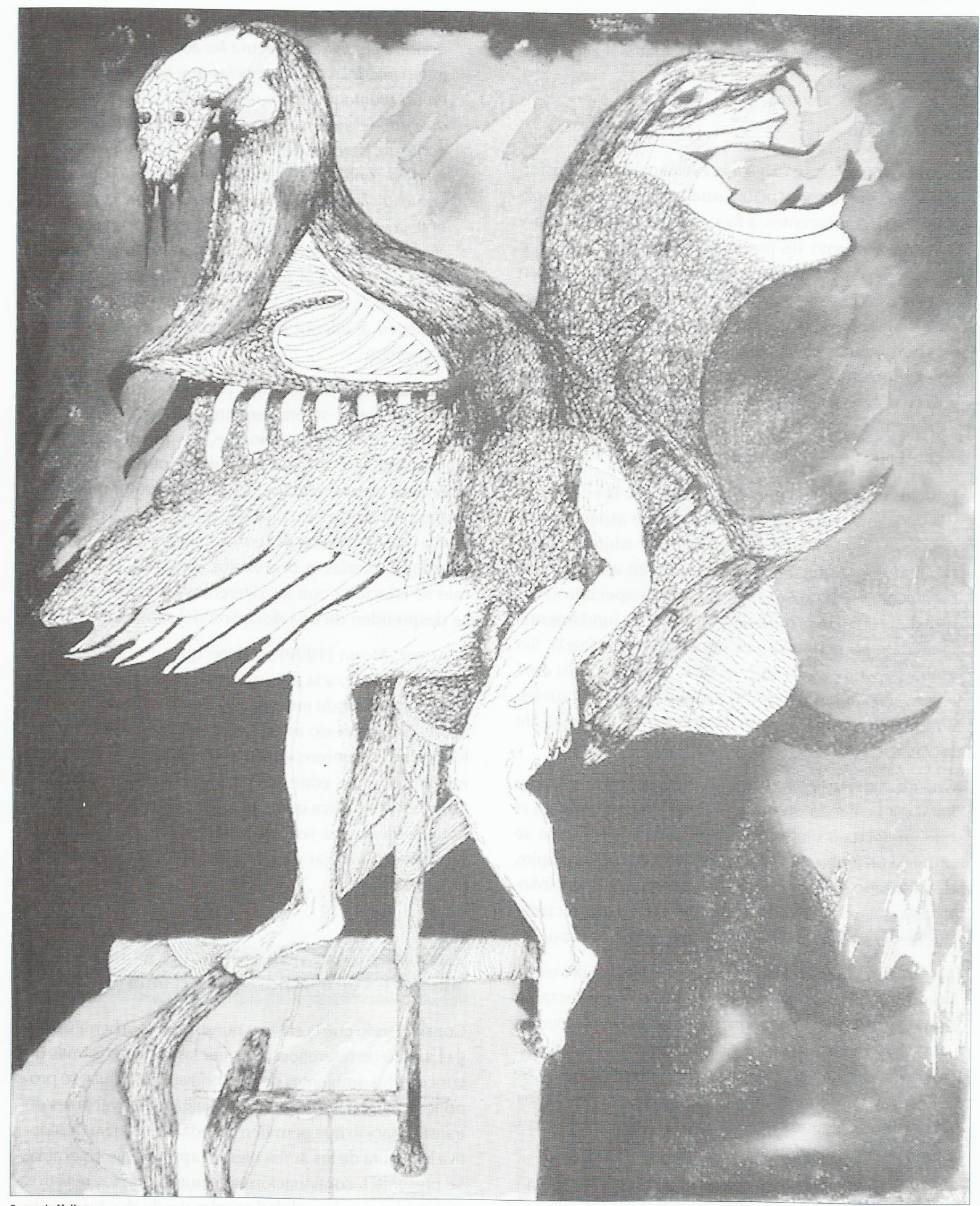

Fernando Molina 
vista: primero, la mirada introspectiva del maestro titular como un observador participante, y segundo, la mirada de un colega como observador no participante.

Las tres primeras tablas corresponden a la posición reflexiva del docente titular, quien indaga sobre la relación entre sujeto, actividad y contexto desde la vivencia del estudiante. Las dos siguientes priorizan tales relaciones desde el accionar del docente titular, leídas por un par académico, que asume el papel de observador no participante.

Institución: IED Antonio Baraya.

Actividad: Ondas lumínicas.

Curso: séptimo.

Fecha: 10 de mayo de 2005.

Maestra titular: Edith C. Negrete.

Observación

... el compromiso de la docente es en doble vía, no solo en impartir instrucciones, sino también en observar detalles sobre cómo los estudiantes relacionan variables que influyen directamente en la caracterización del ambiente de aprendizaje que se dinamiza en la clase de biología ... (Segmento)
Variables de transformación

En las relaciones interpersonales entre estudiantes se evidencia una organización en grupos según intereses que van desde lo personal hasta lo académico, estableciéndose y replanteando normas de convivencia.

En cuanto a la relación estudiante-actividad se trabaja en torno a una puntual: construcción del periscopio. Los estudiantes construyen un modelo y abordan preguntas individualmente y en grupo, posibilitando un aprendizaje significativo y un trabajo colaborativo.

En la relación estudiante-escenario: trabajan de acuerdo con la disponibilidad de recursos físicos: guía o materiales para la construcción del periscopio, en donde manipulan información y contrastan saberes.

En la relación estudiante-contexto, se observan estudiantes motivados y otros que aún no se han entusiasmado por la actividad.
El supuesto del que parte la profesora en el desarrollo del tema es despertar el interés de los estudiantes por aprender, que transforme opiniones personales; esto se evidencia en la argumentación y a la vez brinda elemen- tos para la formación personal como la responsabilidad y la autonomía. En este ambiente de aprendizaje prevalece la relación sujeto-actividad didáctica.

Institución: IED José Martí.

Actividad: Armar palabras.

Curso: segundo.

Fecha: 12 de mayo de 2005.

Maestra titular: Cristina Behrentz P.

\section{Observación}

... la actividad es un concurso en el cual los niños en subgrupos forman palabras con una lotería de letras. Se establecieron reglas del juego en donde cada grupo recibe puntos. En este trabajo se observó lo siguiente:

- La motivación, entendida como participación cuando los niños rápidamente armaban las palabras y se ayudaban.

- La actitud, cuando se gana o se pierde, por ejemplo. La alegría al recibir los puntos y el disgusto de perder.

- La agilidad, la conciencia lingüística de los niños al armar palabras. En el caso de la palabra "perro" unos grupos lo hacían rápidamente, otros escribían "pero"... (Segmento)

\section{Variables de transformación}

Sujeto-actividad didáctica: la intención se logra cuando los niños arman las palabras con emoción y rapidez. Los niños participan, inclusive los que no tienen material se integran a los grupos de forma voluntaria. Los niños se autoevalúan al reconocer sus errores.

- Sujeto-escenario: el salón está organizado en grupos, el material didáctico es elaborado por los padres.

- Sujeto-contexto: los estudiantes tienen un vocabulario escaso, la familia no cuenta con un nivel de escolarización, lo cual dificulta el acompañamiento de los mismos en la actividad.
El ambiente de aprendizaje en el aula es intencionado por el docente según la pretensión que tenga; para el caso, partir de una actividad en donde los estudiantes dinamizan el proceso. 
Institución: Entre Nubes Suroriental.

Actividad: Producción textual: la descripción.

Curso: segundo.

Fecha: 11 de mayo de 2005.

Maestro titular: J. Frank Becerra Martínez.

\section{Observación}

Es la 1:00 p.m, la tarde es bastante fría... El profesor los está esperando, saluda a uno por uno... el profesor pregunta las características de algunos objetos cotidianos. Alexánder lee en voz alta, lo hace muy bien, los compañeros lo aplauden y hace un gesto gracioso.

Algunos niños empiezan a distraerse, el profesor finalmente copia en el tablero lo que acaba de decir, lo que hace que la atención se centre nuevamente en la actividad. Ahora van a describir un perro, dice el profesor. Daniela escribe "un perro es chiquito y también es bravo con cara fea con cancha y tienen dientes muy ágiles y muerden muy duro. Los más grandes son muy bravos y más comen, cuando los molestan no hacen sino salir a morderlo a uno" (tomado textualmente del cuaderno de la niña ) ... el profesor escribe la tarea en el tablero y les dice que después de copiarla guarden el cuaderno... son las $2: 15$ p. m., se da por terminada la actividad y finaliza la observación. (Segmentos)
Variables de transformación

En la clase se ponen en evidencia algunos elementos, especialmente las formas de trabajo y las relaciones sujeto-actividad didáctica en cuanto intención apropiación y recurso (...) los estudiantes levantan la mano para participar.(...) de alguna manera está indicando la dinámica de este grupo. Donde la actividad está planteada para favorecer el trabajo individual y la socialización del mismo (...) atiende uno por uno, pero se detiene con interés en algunos (...) los que necesitan refuerzo constante (según el maestro) (...) es una estrategia utilizada en este caso para "asegurar" apropiación (desde el docente) de la actividad.
Los ambientes de aprendizaje en el aula tienen la característica de poner en interacción e interrelación tres elementos: actores, actividad didáctica y contexto, pero en algunos casos o en un momento específico, uno o dos de estos parecen valorar los otros y da la impresión de ser el que está dando cuenta de los ambientes de aprendizaje en el aula; es el caso de esta vivencia, donde la actividad didáctica prima sobre las demás.

Institución: IED Antonio Baraya.

Actividad: Práctica sobre las propiedades de la luz.

Curso: 702.

Fecha: 28 de abril de 2005.

Observador no participante: Bibiana Quiroga Pérez.

Docente titular: Edith Constanza Negrete Soler.

Los estudiantes llegan al laboratorio donde son esperados por el docente quien luego del saludo inicia la clase expresando $i \mathrm{~A}$ quién le corresponde la exposición de hoy? $\mathrm{Al}$ ver que la estudiante encargada de tal temática no asistió, plantea los aspectos principales sobre la luz. En seguida los estudiantes se organizan en grupos $y$, dadas las indicaciones respectivas, desarrollan la aplicación de lo visto a partir de una experiencia práctica de laboratorio...

La docente posibilita la aclaración de inquietudes a través de su acompañamiento en cada grupo de trabajo...Terminado el ejercicio se plantea la actividad para la casa. (Segmento)
Estudiantes: se evidencian unas "alianzas" previamente establecidas, fundamentalmente por afectos de género que son comunicadas no solo de forma verbal sino también gestual lo que corresponde a un argot construido y comprendido por ellos.

- Docente-estudiantes: en ocasiones se intenta propiciar una relación menos formal por parte de los estudiantes pero se supedita a términos académicos y de cumplir con la clase por parte del docente.

- Sujeto-actividad: resulta motivante el trabajo práctico en tanto da la posibilidad de manipular y evidenciar empíricamente unos referentes teóricos que por sí solos no tendrían sentido.

- Sujeto-escenario: el aprovechamiento de los diferentes recursos didácticos y del espacio físico del laboratorio se convirtieron en una posibilidad de manipulación a través de la cual se vivencia el conocimiento.

- Sujeto-contexto: los intereses de los estudiantes son un referente importante al considerar la pertinencia de los contenidos en tanto resultan limitantes frente a una verdadera significación. 
Dentro del aula se evidenciaron relaciones, situaciones y acciones que tienen ver con las múltiples maneras de aprender, interactuar y enseñar propios de una dinámica que se construye en conjunto (docentes-estudiantes). El interés particular del docente por ofrecer, a su manera de ver, el mejor ambiente de aprendizaje resulta indispensable cuando con sus actitudes, gestos y palabras expresa el verdadero sentir de su hacer. Por su parte, el estudiante, más allá de considerar la clase como posibilidad o no de formación, la asume como propia en términos de encuentro, creación y búsqueda de referentes significativos en su vivir.

Institución: IED Antonio Baraya.

Actividad: Sentido de la vista.

Curso: séptimo.

Fecha: 27 de abril de 2005.

Observador no participante: Mireya Garzón.

Docente titular: Edith Negrete.

Observación

Se inicia dentro del laboratorio como escenario de trabajo, realizando algunas preguntas e involucrando a los estudiantes entre 14 y 16 años de edad, de manera informal, en el desarrollo del tema central de la clase...

Se evidencian diversas actitudes y aptitudes de los estudiantes hacia la clase, entre las cuales, apatía, desconcentración, recocha, pero el compromiso de la docente es tal que los ve no como obstáculos para el desarrollo de la actividad sino que los convierte en puntos a su favor, logrando en ellos la atención y apropiación de la actividad...

Los recursos didácticos son aprovechados de tal forma que se posibilita la construcción efectiva del conocimiento. (Segmento)

En cuanto a los ambientes de aprendizaje en el aula, se evidencian varios elementos como: escenario, sujeto, actividad didáctica y contexto. Estos se interrelacionan e interactúan entre sí durante el desarrollo de una actividad puntual como la descrita anteriormente; aportan y enriquecen la práctica pedagógica docente y la construcción de conocimiento; para el docente son de interés particular la actividad didáctica y la relación sujeto-actividad.

En la observación participante el maestro titular toma distancia por momentos en su trabajo cotidiano para registrar las vivencias de su aula. En el diario de campo quedan consignados comentarios y testimonios como "... Alexánder lee en voz alta, lo hace muy bien, los compañeros lo aplauden y él hace un gesto gracioso..."; "... cada grupo recibe puntos después de armar palabras..."; se evidencia un sinnúmero de interacciones que comúnmente pasan desapercibidas como la participación y el impacto de lo que el maestro hace y dice sobre sus estudiantes, entre otras.

La mirada que se tiene de los ambientes de aprendizaje en el aula es que la relación más incidente es la que se establece entre el docente y la actividad didáctica.

\section{Variables de transformación}

Se evidencian algunos indicadores cualitativos, de los cuales podemos enunciar:

- Relaciones interpersonales: se presenta una información general para una posterior organización grupal.

- Sujeto-actividad didáctica: durante el desarrollo de la actividad se explicita la intención, apropiación y producción a partir de la participación y vivencia del aprendizaje.

- Sujeto-escenario: este último se convierte en un recurso en tanto se aprovecha como fuente de información y complementariedad.

- Sujeto-contexto: hay una pertinencia del saber en tanto resulta aplicable a la realidad del estudiante.

¿Qué connotación tiene para el docente la relación que se da entre estudiante y estudiante como constructor de conocimiento?

En la observación no participante se evidencia la actitud de los estudiantes frente a la actividad propuesta por el docente, además se da cuenta de "unas alianzas previamente establecidas por afectos de género..." que resaltan el interés de los actores por optimizar el escenario como fuente de saber y hacer en contexto. El ambiente de aprendizaje en el aula resulta ser una construcción dinámica y compleja entre estudiantes y maestro a la base de una actividad didáctica.

\section{Reflexión final}

Esta propuesta busca transformar el ambiente de aprendizaje, reflexionar sobre los principios que orientan la escuela, las expectativas, intenciones y deseos del maestro y del estudiante. Además, comprender y diferenciar el proceder en el aula que permita construir significativamente procesos de conocimiento y transformar las relaciones con el otro y con el entorno. 
BECERRA, J. et ál. Un acercamiento a la concepción de escuela en los niños y niñas del CED Santa Rita Suroriental, jornada tarde. Investigación realizada en el año 2000. Sin publicar.

CÁRDENAS, D. y PARRA, M. 2000. Recuperación del sentido de lo humano para generar un ambiente democrático en nuestras escuelas. Memorias II Encuentro de maestros y comunicación de experiencias: "¿Es posible otra escuela?". Bogotá: Corporación Escuela Pedagógica Experimental.

CASTILLO, G. y otros 2002. Colectivo de Investigación Muchl Kaambal. III Encuentro Iberoamericano de colectivos escolares y redes de maestros que hacen investigación en la escuela. Santa Marta.

HARGREAVES, A. 1996. Profesorado, cultura y posmodernidad. Cambian los tiempos, cambia el profesorado. Madrid: Morata.

LÓPEZ RAYÓN, A. 2004. Ambientes de aprendizaje. Documento sin publicar. Bogotá.

MATURANA, H. 1997. El sentido de lo humano. Santiago de Chile: Dolmen.

MINISTERIO DE EDUCACIÓN NACIONAL. 1998. Ética y valores humanos. Lineamientos curriculares. Bogotá: Cooperativa Editorial Magisterio.

MORIN, E. 1999. Los siete saberes necesarios para la educación del futuro. Unesco.

PÉREZ GÓMEZ, Á. 1998. La cultura escolar en la sociedad neoliberal. Madrid: Morata.

RODRÍGUEZ, R. 1999. "Investigación y currículo". Revista Ciencia y Tecnología, No. 6. Bogotá: Universidad Pedagógica Nacional.

SALINAS, J. 1997. "Nuevos ambientes de aprendizaje para una sociedad de la información". Revista Pensamiento Educativo. Chile: PUC, p. 20.

SÁNCHEZ, M. Ambientes de aprendizaje con robótica pedagógica. Bogotá. Artículo de Internet.

SECRETARÍA DE EDUCACIÓN DE BOGOTÁ. 2000. Hacia una cultura de la evaluación para el siglo XXI. Taller sobre Evaluación de Competencias Básicas. Bogotá.

SEGURA, D. 1999. La construcción de la confianza. Una experiencia en proyectos de aula. Colección Polémica Educativa. Bogotá: Escuela Pedagógica Experimental, IDEP.

2005. "Proyectos de aula". Revista Magisterio, 2, abrilmayo. Bogotá.

SUÁREZ, R. 2002. Educación y sociedad. La educación. México: Trillas.

TORRES, E. 1999. Fantasmas y utopías. Ponencia presentada en el VIII Foro Pedagógico. Compensar. Bogotá.

\section{Diálogo del conocimiento}

En las conversaciones que Don Juan sostenía con Carlos Castaneda, siempre utilizaba la expresión "hombre de conocimiento", pero nunca le explicaba qué quería decir. Después de mucha insistencia le hizo saber que para ser un hombre de conocimiento era necesario vencer cuatro enemigos naturales: el miedo, la claridad, el poder y la vejez. Para Carlos Castaneda tal vez esta enseñanza respondió a su pregunta.

Se me antoja pensar que la pregunta ¿qué significa ser un hombre de conocimiento? está formulada en este rollo de maneras distintas, y de distintas maneras en él se despliega. Para ello, sin temor a equívocos, los maestros que lo elaboran han tenido que vencer dos miedos: uno, el que genera el hacerse evidente para el otro en el diálogo, y otro, el que genera el encuentro con la escritura.

Desde el primero, la insularidad que el maestro yergue como su más estimada seguridad, en la que los textos escolares y los planes de estudio son sus adalides, se deconstruye, se anega..., en el encuentro con el otro, que cual náufrago relata las aventuras de su soledad pedagógica. Emergen certezas, grandes hallazgos, experiencias inéditas, extravíos, personajes memorables de los cuales heredaron baratijas de espejos, en los que ninguno puede ver su propio rostro. ¿Quién está ahí, quién es ese que mira y no responde? Con el diálogo la baratija se rompe, quien responde ya no es el espejo, escucha otras voces, otras aventuras; ha llegado a un continente de náufragos y... comienza otra aventura. Sin saber cómo, sin dejar de ser lo que ha sido, las voces que escucha, cada vez más claras, cada vez más audibles, hablan de lo que siente, de lo que piensa, de lo que sueña... a solas. Sus relatos se vuelven plurales, su aventura se vuelve una experiencia compartida. Y sin darse cuenta, se topa con el segundo enemigo: la escritura.

¿Qué hemos hecho? ¿Cómo lo hemos hecho? ¿Por qué hemos hecho esto y no otra cosa? Seguramente estas y otras preguntas transforman la aventura individual de contar las anécdotas por la aventura colectiva de trascender la insularidad y comprender cómo es esto posible. Este es, creo, el sentido de Los ambientes de aprendizaje en el aula: la experiencia de un grupo de maestros que decidieron dejar a un lado las certezas de un otro sin rostro, para construir su propio rostro, para, mirando al otro, desde lo que escribe, transformar su propia práctica.

Steiner Valencia Vargas 